\title{
Linking Type la Supernova Progenitors and Their Resulting Explosions
}

\section{Citation}

Foley, Ryan J., Joshua D. Simon, Christopher R. Burns, Avishay Gal-Yam, Mario Hamuy, Robert P. Kirshner, Nidia I. Morrell, Mark M. Phillips, Gregory A. Shields, and Assaf Sternberg. 2012. "LINKING TYPE la SUPERNOVA PROGENITORS AND THEIR RESULTING EXPLOSIONS." The Astrophysical Journal 752 (2): 101. https://doi.org/10.1088/0004-637x/752/2/101.

\section{Permanent link}

http://nrs.harvard.edu/urn-3:HUL.InstRepos:41399792

\section{Terms of Use}

This article was downloaded from Harvard University's DASH repository, and is made available under the terms and conditions applicable to Other Posted Material, as set forth at http:// nrs.harvard.edu/urn-3:HUL.InstRepos:dash.current.terms-of-use\#LAA

\section{Share Your Story}

The Harvard community has made this article openly available. Please share how this access benefits you. Submit a story.

Accessibility 


\title{
LINKING TYPE Ia SUPERNOVA PROGENITORS AND THEIR RESULTING EXPLOSIONS
}

\author{
Ryan J. Foley ${ }^{1,8}$, Joshua D. Simon ${ }^{2}$, Christopher R. Burns ${ }^{2}$, Avishay Gal-Yam ${ }^{3}$, Mario Hamuy ${ }^{4}$, Robert P. Kirshner ${ }^{1}$, \\ Nidia I. Morrell ${ }^{5}$, Mark M. Phillips ${ }^{5}$, Gregory A. Shields ${ }^{6}$, and Assaf Sternberg ${ }^{7,9}$ \\ ${ }^{1}$ Harvard-Smithsonian Center for Astrophysics, 60 Garden Street, Cambridge, MA 02138, USA; rfoley @ cfa.harvard.edu \\ ${ }^{2}$ Observatories of the Carnegie Institution for Science, 813 Santa Barbara Street, Pasadena, CA 91101, USA \\ ${ }^{3}$ Benoziyo Center for Astrophysics, Faculty of Physics, Weizmann Institute of Science, Rehovot 76100, Israel \\ ${ }^{4}$ Departamento de Astronomía, Universidad de Chile, Casilla 36-D, Santiago, Chile \\ ${ }^{5}$ Las Campanas Observatory, Carnegie Observatories, Casilla 601, La Serena, Chile \\ ${ }^{6}$ Department of Astronomy, University of Texas, Austin, TX 78712, USA \\ ${ }^{7}$ Max-Planck-Institut für Astrophysik, Karl-Schwarzschild-Straße 1, 85741 Garching, Germany \\ Received 2012 March 13; accepted 2012 April 14; published 2012 May 31
}

\begin{abstract}
Comparing the ejecta velocities at maximum brightness and narrow circumstellar/interstellar Na D absorption line profiles of a sample of 23 Type Ia supernovae (SNe Ia), we determine that the properties of SN Ia progenitor systems and explosions are intimately connected. As demonstrated by Sternberg et al., half of all SNe Ia with detectable Na $\mathrm{D}$ absorption at the host-galaxy redshift in high-resolution spectroscopy have Na D line profiles with significant blueshifted absorption relative to the strongest absorption component, which indicates that a large fraction of SN Ia progenitor systems have strong outflows. In this study, we find that SNe Ia with blueshifted circumstellar/interstellar absorption systematically have higher ejecta velocities and redder colors at maximum brightness relative to the rest of the SN Ia population. This result is robust at a 98.9\%-99.8\% confidence level, providing the first link between the progenitor systems and properties of the explosion. This finding is further evidence that the outflow scenario is the correct interpretation of the blueshifted $\mathrm{Na} \mathrm{D}$ absorption, adding additional confirmation that some SNe Ia are produced from a single-degenerate progenitor channel. An additional implication is that either SN Ia progenitor systems have highly asymmetric outflows that are also aligned with the SN explosion or SNe Ia come from a variety of progenitor systems where SNe Ia from systems with strong outflows tend to have more kinetic energy per unit mass than those from systems with weak or no outflows.
\end{abstract}

Key words: distance scale - dust, extinction - supernovae: general

Online-only material: color figures

\section{INTRODUCTION}

Type Ia supernovae (SNe Ia) appear to come from carbon-oxygen white dwarfs (WDs) in a binary system (Hoyle $\&$ Fowler 1960); however, it is still unknown if they result from the merger of two WDs (the "double-degenerate" scenario; Iben \& Tutukov 1984; Webbink 1984) or a single WD accreting material from a non-degenerate companion (the "single-degenerate" scenario; Whelan \& Iben 1973; Iben \& Tutukov 1996). Howell (2011) and Wang \& Han (2012) present recent reviews on the subject. With the exception of the recently discovered, nearby SN Ia 2011fe (Li et al. 2011; Nugent et al. 2011; Bloom et al. 2012; Brown et al. 2011; Chomiuk et al. 2012; Margutti et al. 2012), there are only weak constraints on the nature of the binary companion for individual SNe Ia (e.g., Maoz \& Mannucci 2008; Foley et al. 2010, 2012; Edwards et al. 2012; Schaefer \& Pagnotta 2012), and even then it is difficult to completely exclude scenarios for subsamples of SNe Ia. Similarly, there are several different models for SN Ia explosions (e.g., Nomoto et al. 1984; Woosley et al. 1986; Khokhlov 1991; Hillebrandt \& Niemeyer 2000; Gamezo et al. 2005; Pakmor et al. 2010; van Kerkwijk et al. 2010; Sim et al. 2010) and limited observational constraints (Blondin et al. 2011). This is particularly troubling since measurements of SNe Ia were used to discover the accelerating expansion of the universe (Riess et al. 1998; Perlmutter et al. 1999) and continue to be exquisite tools for constraining cosmological parameters (Wood-Vasey et al. 2007; Riess et al.

\footnotetext{
8 Clay Fellow.

9 Minerva Fellow.
}

2007; Freedman et al. 2009; Hicken et al. 2009b; Kessler et al. 2009; Amanullah et al. 2010; Conley et al. 2011; Suzuki et al. 2012).

The recent observations of time variable, narrow $(\Delta v<$ $100 \mathrm{~km} \mathrm{~s}^{-1}$; resulting from either circumstellar or interstellar material in the host galaxy) $\mathrm{Na} \mathrm{D}$ absorption in several SNe Ia (SNe 2006X, 1999cl, and 2007le; Patat et al. 2007b; Blondin et al. 2009; Simon et al. 2009) are perhaps the best evidence that at least some SN Ia progenitor systems have strong outflows - the hallmark of certain classes of single-degenerate progenitor systems. In the two cases with high-resolution spectroscopy, the variable component of the $\mathrm{Na} \mathrm{D}$ absorption profile was blueshifted relative to the strongest component of the profile. Using a large sample of SNe Ia with single-epoch highresolution spectroscopy, Sternberg et al. (2011, hereafter S11) determined that about half of all SNe Ia with Na D absorption have blueshifted $\mathrm{Na} \mathrm{D}$ absorption relative to the position of the strongest absorption (12/22 SNe Ia). In the same sample, only a quarter of SNe Ia $(5 / 22)$ have redshifted absorption, while the remaining quarter $(5 / 22)$ have single or symmetric absorption profiles. If the absorption were the result of only interstellar gas, we would expect the fraction of blueshifted and redshifted profiles to be equal. S11 concluded that the difference in these numbers was likely the result of a large fraction of $\mathrm{SNe}$ Ia ( $25 \%$ of $\mathrm{SNe}$ Ia with detected $\mathrm{Na} \mathrm{D}$ absorption) coming from progenitor systems with strong outflows.

The first SN Ia with identified variable Na D was SN 2006X (Patat et al. 2007b). This SN was also exceptional in its high ejecta velocity and high reddening (Wang et al. 2008). With only the detection of variable Na D in SN 2006X and the 
non-detection of variable Na D in the low-velocity SN 2007af, Simon et al. (2007) hypothesized that variable $\mathrm{Na} \mathrm{D}$ was exclusive to $\mathrm{SNe}$ Ia with high ejecta velocities. Since then, detections of variable $\mathrm{Na} \mathrm{D}$ in SNe 1999cl (Blondin et al. 2009) and 2007le (Simon et al. 2009) have been made_-both SNe Ia with relatively high $\mathrm{Si}$ II $\lambda 6355$ velocities, $v_{\mathrm{Si} \text { II }}$, near maximum brightness.

Additionally, ejecta velocity provides insight into the explosion. Directly, the ejecta velocity (up to viewing angle effects of asymmetric explosions) probes the kinetic energy per unit mass. If one assumes that all SNe Ia have approximately the same total mass (e.g., Mazzali et al. 2007) and relatively small asymmetries (e.g., Leonard et al. 2005), then ejecta velocity is a good tracer of the kinetic energy of the explosion.

The strong correlation seen between the velocity gradient of the Si II $\lambda 6355$ feature near maximum brightness of an SN Ia and the relative redshifting of forbidden lines in its nebular spectrum (Maeda et al. 2010) places strong constraints on explosion models. The early-time velocity measures the characteristics of the outer layers of the $\mathrm{SN}$, while the late-time velocity measures the characteristics of the inner ejecta. The connection can be interpreted as the result of an asymmetric explosion, where different viewing angles result in different velocities at early and late times (Maeda et al. 2010). This asymmetric explosion interpretation can further explain the relation between SN Ia ejecta velocity (and velocity gradient) and intrinsic color (Foley \& Kasen 2011; Maeda et al. 2011).

While ejecta velocity probes the characteristics of the SN explosion, the line profile of narrow $\mathrm{Na} \mathrm{D}$ is influenced by the progenitor environment. There has been tenuous evidence that $\mathrm{SNe}$ Ia with variable $\mathrm{Na} \mathrm{D}$ tend to have higher-velocity ejecta. To determine if this trend is happenstance, we use the large Foley et al. (2011, hereafter FSK11) collection of maximumbrightness $\mathrm{Si}$ II $\lambda 6355$ velocities for $\mathrm{SNe} \mathrm{Ia}, v_{\mathrm{Si} \text { II }}^{0}$, supplemented by additional data presented here to both expand the S11 sample and assign ejecta velocities to that expanded sample.

The rest of this paper is structured as follows. In Section 2, we present new data to expand the FSK11 and S11 samples and then match the expanded samples. In Section 3, we examine the properties of the matched sample, finding that $\mathrm{SNe}$ Ia with blueshifted $\mathrm{Na} \mathrm{D}$ line profiles tend to have higher ejecta velocities and redder colors than typical SNe Ia. We discuss this result and conclude in Section 4.

\section{DATA}

In this section, we construct our sample. We begin with the S11 sample of SNe Ia and add to it additional SNe Ia with new high-resolution spectroscopy. We discuss the FSK11 sample of $\mathrm{SNe} \mathrm{Ia}$, and add $\mathrm{SNe}$ Ia with new photometry and lowresolution spectroscopy to the sample. We exclude all $\mathrm{SNe}$ Ia with $\Delta m_{15}(B)>1.5 \mathrm{mag}$ since we cannot determine an accurate $v_{\mathrm{Si} \text { II }}^{0}$ for these $\mathrm{SNe}$. Our final sample, the F12 sample, is comprised of all spectroscopically normal SNe Ia with highresolution spectroscopy and a measurement of $v_{\mathrm{Si} \text { II }}^{0}$.

\subsection{High-resolution Spectroscopy}

S11 present a sample of $35 \mathrm{SNe}$ Ia with high-resolution spectroscopy primarily obtained with the Keck, Magellan, and VLT telescopes. For all spectra with any narrow $\mathrm{Na} \mathrm{D}$ absorption at the recession velocity of the host galaxy, a zero velocity for the system was assigned to the strongest $\mathrm{Na} \mathrm{D}$ component. The $\mathrm{SNe}$ were then classified as having blueshifted, redshifted, single, or symmetric profiles. If, for example, the $\mathrm{Na} \mathrm{D}$ profile had additional absorption offset primarily to the blue of the zero-velocity component, it was classified as Blueshifted. Depending on the relative strengths of circumstellar and interstellar absorption, it is possible in principle that some SNe Ia with strong-outflow progenitor systems could appear as Redshifted, Single, or (possibly) Symmetric. However, in a large sample of SNe with circumstellar material (CSM), the Blueshifted objects should outnumber objects in the Redshifted sample. Of the $35 \mathrm{SNe}$ Ia, S11 find that 12,5 , and $5 \mathrm{SNe}$ Ia have blueshifted, redshifted, and single or symmetric narrow $\mathrm{Na} \mathrm{D}$ absorption profiles, respectively, while 13 have no detected $\mathrm{Na} \mathrm{D}$ absorption.

Using additional data, we modify the S11 sample. Specifically, we exclude SN 2008ge (which had no Na D absorption) because of its known peculiarities (Foley et al. 2010). S11 decided not to include some older SNe Ia in their sample because of a possible publication bias (i.e., SNe without detected or extraordinary $\mathrm{Na} \mathrm{D}$ line profiles may not have ever been published). Since we are comparing velocities within our sample, this publication bias should not affect us significantly. We therefore add SN 1986G (Rich 1987; D’Odorico et al. 1989), which has a blueshifted Na D line profile, and SN 2001el (Sollerman et al. 2005), which has a redshifted Na D line profile. Although SN 2000cx has high-resolution spectra (showing no Na D absorption; Patat et al. 2007a), we do not add this SN to the S11 sample because of its peculiar nature (Li et al. 2001).

We also consider SN 1994D (Ho \& Filippenko 1995; King et al. 1995; Patat et al. 1996). Its Na D line profile is complicated by its very low recession velocity. There are three groups of absorption lines at $\sim 0,200-250$, and $700 \mathrm{~km} \mathrm{~s}^{-1}$, respectively. The feature at $700 \mathrm{~km} \mathrm{~s}^{-1}$ is clearly a single absorption line. If one interprets the features at $\sim 200-250 \mathrm{~km} \mathrm{~s}^{-1}$ as being associated with the Milky Way, then SN 1994D would be classified as a Single object. However, if those features are associated with the SN or its host galaxy, then it would be classified as Blueshifted. In the S11 sample, there were no features beyond $\sim 150 \mathrm{~km} \mathrm{~s}^{-1}$ relative to the strongest absorption feature, so a $\sim 450-500 \mathrm{~km} \mathrm{~s}^{-1}$ offset would be an extreme outlier. Nonetheless, we do not claim to fully understand the characteristics of potential SN Ia progenitor outflows. We consider the possibility of SN 1994D being a Blueshifted SN as low, and for our main analysis we classify it as Single. However, we also performed the analysis with SN 1994D classified as Blueshifted. The main results are not significantly affected by this choice.

We add new high-resolution spectra of six SNe Ia obtained with the High-Resolution Spectrograph (HRS; Tull 1998) on the Hobby-Eberly Telescope (HET) in 2001 and 2002. The HRS observations used the $600 \ell \mathrm{mm}^{-1}$ grating centered at $6302 \AA$, providing wavelength coverage from 5300 to $7300 \AA$ at $R=30,000$, and were made through a $3^{\prime \prime}$ diameter fiber.

The HET data were reduced in IRAF with the ECHELLE package using standard procedures. Wavelength calibration was performed using spectra of a Th-Ar comparison lamp obtained immediately before or after the SN observations. The Na D emission lines in the HRS flat-field lamps were masked out before flat-fielding. Emission from the night sky was removed by subtracting the spectrum obtained through a sky fiber after scaling to match the sky emission line intensities in the SN spectrum (this scaling matches the relative throughputs of the science and sky fibers derived from internal flat-field frames when available). The normalized-flux spectra near the hostgalaxy $\mathrm{Na} \mathrm{D}$ doublet are presented in Figure 1. 


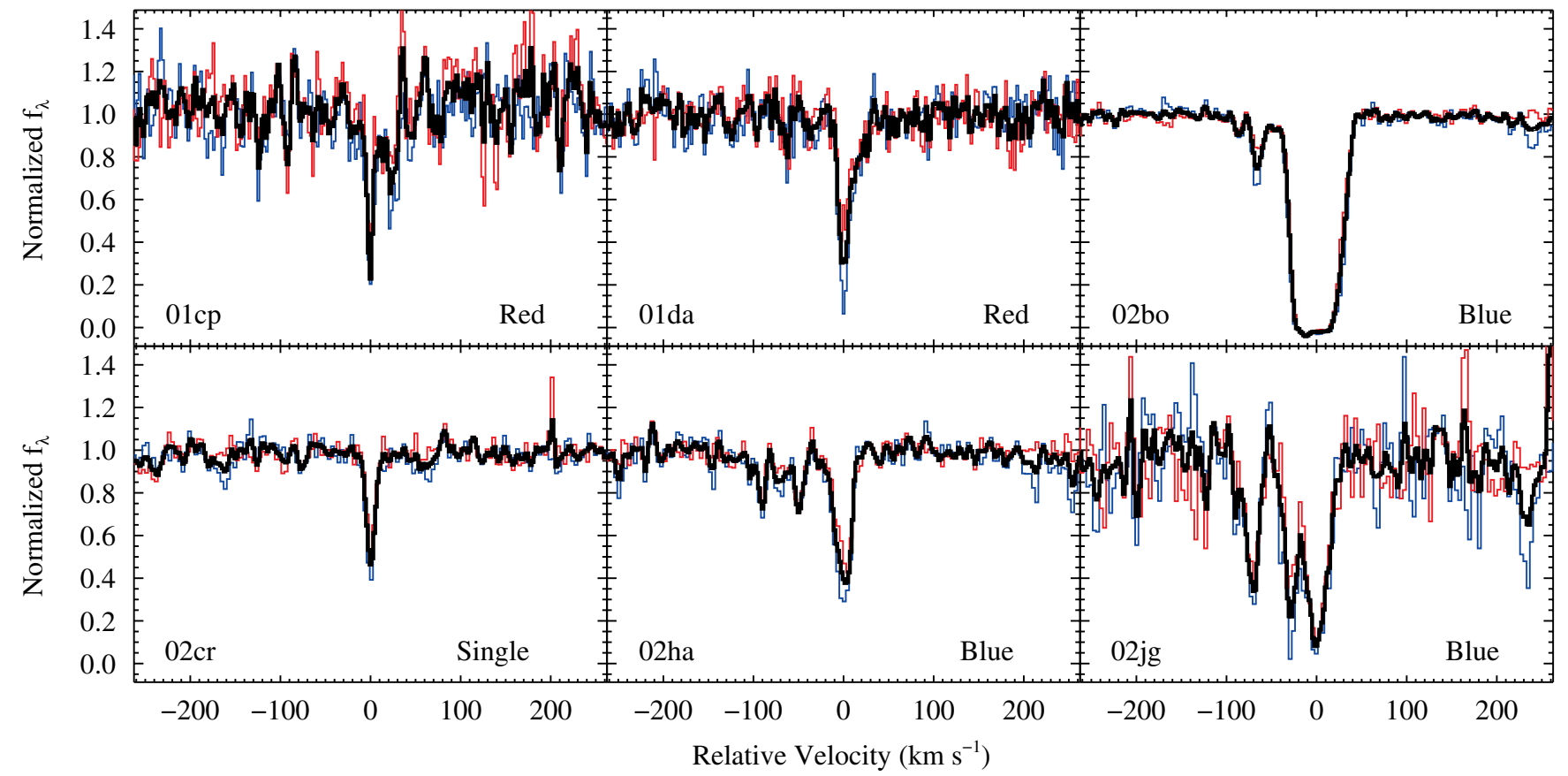

Figure 1. High-resolution HET spectra of SNe 2001cp, 2001da, 2002bo, 2002cr, 2002ha, and 2002jg. The spectra are presented in normalized flux units and only the region near $\mathrm{Na} \mathrm{D}$ is shown. The data are presented on a velocity scale where the zero velocity corresponds to the strongest absorption. The red and blue spectra are the individual Na D1 and Na D2 components, respectively, while the black spectra represent the combination of the two components. We label each SN and its profile designation in each panel.

(A color version of this figure is available in the online journal.)

We report the properties of the expanded high-resolution SN Ia sample in Table 1 .

\subsection{Photometry}

FSK11 present $1630 v_{\text {Si II }}$ measurements for $255 \mathrm{SNe}$ Ia (Blondin et al. 2012). Using a family of functions, they were able to estimate $v_{\mathrm{Si} \text { II }}$ at maximum brightness, $v_{\mathrm{Si} \text { II }}^{0}$, for $\mathrm{SNe}$ Ia with moderate decline rates and $v_{\mathrm{Si} \text { II }}$ measured within about a week of maximum brightness. This large sample provides the reference sample for this work. Although there are potential differences between this sample and the underlying SN Ia sample, the size of the sample provides a reasonable baseline. To further increase the overlap between the expanded S11 sample and the FSK11 sample, here we provide new photometry, spectroscopy, and/or derived values for seven SNe Ia.

FSK11 presented several light-curve-derived SN quantities $\left(t_{\max }, B_{\max }-V_{\max }\right.$, and $\left.\Delta m_{15}(B)\right)$ that are critical for measuring $v_{\mathrm{Si} \text { II }}^{0}$. The light curves and original derived quantities were part of the CfA3 (Hicken et al. 2009a) and LOSS (Ganeshalingam et al. 2010) data sets. Ganeshalingam et al. (2010) also presented light-curve parameters for SNF20080514-002, which we use for this study. Foley et al. (2012) presented light-curve parameters for SN 2009ig, which we also use here. For SN 2009nr, we use the light-curve properties reported by Khan et al. (2011). Light curves of SNe 2007on, 2008C, 2008fp, 2008hv, and 2008ia were presented in Stritzinger et al. (2011); we derive light-curve parameters for these $\mathrm{SNe}$ by fitting the light curves with the SNooPy Python package (Burns et al. 2011). We have corrected all measurements of $B_{\max }-V_{\max }$ for Milky Way extinction. All light-curve parameters are presented in Table 2.

Additionally, SNe 2009le and 2010A were followed by the FLWO $1.2 \mathrm{~m}$ telescope at Mount Hopkins. Their light curves will be published as part of the CfA4 data set (M. Hicken et al., submitted), but their light-curve parameters are presented here. Again, the light curves were also fitted with SNOoPy, and their light-curve parameters are listed in Table 2.

\subsection{Low-resolution Spectroscopy}

In addition to the new light-curve parameters listed above, we present spectroscopic parameters here, specifically $v_{\text {Si II }}$ and $v_{\mathrm{Si} \text { II }}^{0}$. We use the Foley et al. (2012) spectroscopic measurements for SN 2009ig. SN 2008 C previously had sufficient light curves to determine a time of maximum brightness (FSK11) but not a decline rate or $B_{\max }-V_{\max }$. With additional photometric data, we were able to measure those quantities, but the measurement of $v_{\mathrm{Si} \text { II }}^{0}$ remains unchanged from that of FSK11. For the remaining $\mathrm{SNe}$, we discuss the data acquisition and reduction below.

Additional low-resolution spectra were obtained with a variety of instruments on several telescopes. Specifically, we obtained data using the FAST spectrograph (Fabricant et al. 1998) on the FLWO $1.5 \mathrm{~m}$ telescope, the WFCCD spectrograph mounted on the Du Pont $2.5 \mathrm{~m}$ telescope, the IMACS spectrograph (Dressler et al. 2006) mounted on the Magellan Baade $6.5 \mathrm{~m}$ telescope, and the LDSS-3 spectrograph (Allington-Smith et al. 1994) mounted on the Magellan Clay $6.5 \mathrm{~m}$ telescope. Specifics for each spectrum are presented in Table 2 .

Standard CCD processing and spectrum extraction of the lowresolution data were accomplished with IRAF. The data were extracted using the optimal algorithm of Horne (1986). Loworder polynomial fits to calibration-lamp spectra were used to establish the wavelength scale, and small adjustments derived from night-sky lines in the object frames were applied. The IMACS and LDSS3 spectra were reduced with IRAF routines as described by Hamuy et al. (2006). For the remaining spectra, we employed our own IDL routines to flux calibrate the data 
Table 1

Properties of the Expanded High-resolution SN Ia Sample

\begin{tabular}{|c|c|c|c|c|c|c|}
\hline $\mathrm{SN}$ & UT Date & Phase $^{\mathrm{a}}$ & $\begin{array}{c}\Delta m_{15}(B) \\
(\mathrm{mag})\end{array}$ & $\begin{array}{c}B_{\max }-V_{\max } \\
(\operatorname{mag})\end{array}$ & $\begin{array}{c}v_{\mathrm{Si} \text { II }}^{0} \\
\left(10^{3} \mathrm{~km} \mathrm{~s}^{-1}\right)\end{array}$ & $\begin{array}{l}\text { Host } \\
\text { Type }\end{array}$ \\
\hline \multicolumn{7}{|c|}{ Blueshifted } \\
\hline 2002 bo & 2002 Mar 21.3 & $-2.4(0.7)$ & $1.24(0.05)$ & $0.40(0.04)$ & $-13.1(0.2)$ & $\mathrm{Sa}$ \\
\hline 2002ha & 2002 Nov $2.6^{\mathrm{c}}$ & $0.2(0.7)$ & $1.35(0.05)$ & $-0.09(0.03)$ & $-11.3(0.2)$ & $\mathrm{Sab}$ \\
\hline $2002 \mathrm{jg}$ & 2002 Nov $30.1^{d}$ & $-1.4(0.1)$ & $1.54(0.04)$ & $0.61(0.03)$ & $\ldots$ & Pair \\
\hline 2007le & 2007 Oct 20.4 & $-5.4(0.4)$ & $1.05(0.04)$ & $0.29(0.04)$ & $-13.3(0.2)$ & $\mathrm{Sc}$ \\
\hline $2008 \mathrm{C}$ & 2008 Jan 17.4 & $16.0(0.3)$ & $1.07(0.03)$ & $0.24(0.03)$ & $-11.3(0.2)$ & $\mathrm{S} 0 / \mathrm{a}$ \\
\hline $2008 \mathrm{dt}$ & 2008 Jul 6.5 & $6.5(3.1)$ & $1.02(0.14)$ & $0.55(0.08)$ & $-14.3(0.3)$ & $\mathrm{S} 0 / \mathrm{a}$ \\
\hline $2008 \mathrm{fp}$ & 2008 Sep 17.3 & $-4.1(0.1)$ & $0.92(0.01)$ & $0.49(0.01)$ & $-10.7(0.2)$ & S0 pec \\
\hline 2009ig & 2009 Oct 16.3 & $39.9(0.1)$ & $0.89(0.02)$ & $0.14(0.04)$ & $-13.5(0.2)$ & $\mathrm{Sa}$ \\
\hline \multicolumn{7}{|c|}{ Redshifted } \\
\hline $2001 \mathrm{da}$ & $2001 \mathrm{Jul} 22.4$ & $4.8(1.3)$ & $1.25(0.05)$ & $0.16(0.03)$ & $-11.5(0.2)$ & $\mathrm{Sab}$ \\
\hline 2001el & 2001 Sep $24.8^{f}$ & $-5.3(0.1)$ & $1.15(0.03)$ & $0.03(0.03)$ & $-11.7(0.2)$ & Scd \\
\hline 2007af & 2007 Apr 8.4 & $24.2(0.4)$ & $1.23(0.05)$ & $0.00(0.03)$ & $-11.0(0.2)$ & Scd \\
\hline 2009le & 2009 Nov 26.0 & $-4.8(0.1)$ & $0.96(0.02)$ & $0.12(0.02)$ & $-12.1(0.2)$ & $\mathrm{Sbc}$ \\
\hline $2010 \mathrm{~A}$ & 2010 Jan 8.0 & $-8.2(0.1)$ & $0.88(0.02)$ & $0.16(0.02)$ & $-10.3(0.2)$ & $\mathrm{Sab}$ \\
\hline \multicolumn{7}{|c|}{ Single/Symmetric } \\
\hline $2002 \mathrm{cr}$ & 2002 May 13.2 & $-1.3(0.5)$ & $1.23(0.03)$ & $-0.05(0.03)$ & $-10.1(0.2)$ & Scd \\
\hline $2006 \mathrm{~cm}$ & 2006 Jun 6.5 & $7.0(0.5)$ & $1.04(0.07)$ & $1.07(0.06)$ & $-11.5(0.2)$ & $\mathrm{Sb}$ \\
\hline $2007 \mathrm{sr}$ & 2008 Jan 17.6 & $33.3(0.3)$ & $1.07(0.07)$ & $0.09(0.06)$ & $-12.9(0.3)$ & Sm pec \\
\hline \multicolumn{7}{|c|}{ No Absorption } \\
\hline $2006 \mathrm{eu}$ & 2006 Sep 10.3 & $2.3(2.2)$ & $1.37(0.14)$ & $0.49(0.04)$ & $-11.4(0.3)$ & $\mathrm{E}$ \\
\hline 2007hj & 2007 Oct 20.3 & $43.8(0.2)$ & $1.97(0.06)$ & $0.12(0.03)$ & $\ldots$ & So \\
\hline 2007on & 2008 Jan 17.3 & $62.0(0.1)$ & $1.64(0.01)$ & $0.12(0.01)$ & $\ldots$ & $\mathrm{E}$ \\
\hline 2008 ia & 2008 Dec 15.2 & $2.0(0.1)$ & $1.35(0.01)$ & $0.00(0.01)$ & $-11.5(0.2)$ & So \\
\hline $2009 \mathrm{nr}$ & 2010 Jan 9.3 & $12.5(0.3)$ & $0.93(0.03)$ & $0.00(0.03)$ & $-9.6(0.2)$ & Scd \\
\hline \multicolumn{7}{|c|}{ Ambiguous ${ }^{g}$} \\
\hline 1994D & 1994 Apr $14^{\mathrm{h}}$ & $23.9(0.4)$ & $1.42(0.01)$ & $-0.04(0.04)$ & $-11.2(0.2)$ & $\mathrm{S} 0^{\mathrm{i}}$ \\
\hline
\end{tabular}

Notes. Uncertainties are listed in parentheses.

a Days since $B$ maximum for high-resolution spectrum.

b Exposure-time-weighted mean observation time from D'Odorico et al. (1989), which presented the highest S/N spectrum. Observations span 1986 May 7.2-11.1.

${ }^{\mathrm{c}}$ Exposure-time-weighted mean observation time. Observations span 2002 October 30-November 6.

${ }^{\mathrm{d}}$ Exposure-time-weighted mean observation time. Observations span 2002 November 28-December 2.

e Date corresponding to the first epoch of spectroscopy from Patat et al. (2007b).

${ }^{\mathrm{f}}$ Exposure-time-weighted mean observation time. Observations span 2001 September 21.2-28.3.

g See the text for discussion of the classification of SN 1994D.

${ }^{\mathrm{h}}$ Date for Ho \& Filippenko (1995) observation, which presented the highest S/N spectrum.

i The host galaxy shows an obvious dust lane in images.

and remove telluric lines using the well-exposed continua of spectrophotometric standards (Wade \& Horne 1988; Foley et al. 2003; Silverman et al. 2012).

All details of the low-resolution spectra are presented in Table 2.

We determine $v_{\mathrm{Si} \text { II }}$ and $v_{\mathrm{Si} \text { II }}^{0}$ for these $\mathrm{SNe}$ using the same methods as Blondin et al. (2006) and FSK11. The FSK11 method does not produce accurate $v_{\mathrm{Si} \text { II }}^{0}$ measurements for $\mathrm{SNe}$ Ia with $\Delta m_{15}(B)>1.5 \mathrm{mag}$. As a result, we do not report $v_{\mathrm{Si} \text { II }}^{0}$ for SNe Ia with $\Delta m_{15}(B)>1.5 \mathrm{mag}$. Our measurements for $v_{\mathrm{Si} \text { II }}$ and $v_{\mathrm{Si} \text { II }}^{0}$ are reported in Table 2.

We present the full cross-matched sample of SNe Ia with both $v_{\mathrm{Si} \text { II }}^{0}$ measurements and high-resolution spectroscopy in Table 1.
The $\mathrm{SNe}$ with both $v_{\mathrm{Si} \text { II }}^{0}$ measurements and high-resolution spectroscopy are designated as the "F12" sample.

\section{RESULTS}

Using the F12 sample, we are able to compare different subsamples to each other as well as to the F12 and FSK11 samples. There are currently only four SNe Ia in the Single/Symmetric subsample (when including SN 1994D). Although this subsample is small, it is large enough to perform basic statistical analyses. Nonetheless, we caution against overinterpretation of the results for the Single/Symmetric subsample and suggest that additional analysis should be performed when the sample sizes 
Table 2

Log of Low-resolution Optical Spectral Observations and Basic Parameters

\begin{tabular}{|c|c|c|c|c|c|c|c|}
\hline $\mathrm{SN}$ & UT Date & Instrument & $\begin{array}{c}\text { Time of } \\
\text { Maximum (MJD) }\end{array}$ & Phase $^{\mathrm{a}}$ & $\begin{array}{c}\Delta m_{15}(B) \\
(\mathrm{mag})\end{array}$ & $\begin{array}{c}v_{\text {Si II }} \\
\left(10^{3} \mathrm{~km} \mathrm{~s}^{-1}\right)\end{array}$ & $\begin{array}{c}v_{\mathrm{Si} \mathrm{II}}^{0} \\
\left(10^{3} \mathrm{~km} \mathrm{~s}^{-1}\right)\end{array}$ \\
\hline 2007on & 2007 Nov 17.2 & IMACS & $54419.9(0.1)$ & $1.3(0.1)$ & $1.64(0.01)$ & $-10.9(0.1)$ & $\ldots()$ \\
\hline $2008 C^{b}$ & 2008 Jan 5.3 & FAST & $54466.1(0.3)$ & $4.1(0.3)$ & $1.07(0.03)$ & $-10.9(0.1)$ & $-11.3(0.3)$ \\
\hline SNF20080514-002 & 2008 May 28.3 & FAST & $54613.0(0.5)$ & $1.3(0.5)$ & $1.39(0.04)$ & $-10.7(0.1)$ & $-10.8(0.2)$ \\
\hline $2008 \mathrm{fp}$ & 2008 Sep 21.4 & WFCCD & $54730.5(0.1)$ & $-0.2(0.1)$ & $0.92(0.01)$ & $-10.7(0.1)$ & $-10.7(0.2)$ \\
\hline 2008hv & 2008 Dec 18.3 & LDSS-3 & $54817.1(0.1)$ & $1.1(0.1)$ & $1.33(0.03)$ & $-10.7(0.1)$ & $-10.8(0.2)$ \\
\hline $2008 \mathrm{ia}$ & 2008 Dec 15.3 & LDSS-3 & $54813.2(0.1)$ & $2.1(0.1)$ & $1.35(0.01)$ & $-11.3(0.1)$ & $-11.5(0.2)$ \\
\hline $2009 \mathrm{ig}^{\mathrm{d}}$ & 2009 Sep 5.4 & LRS & $55080.0(0.1)$ & $-0.4(0.1)$ & $0.89(0.02)$ & $-13.6(0.1)$ & $-13.5(0.2)$ \\
\hline 2009le & 2009 Nov 24.2 & FAST & $55165.9(0.1)$ & $-6.6(0.1)$ & $0.96(0.02)$ & $-12.8(0.1)$ & $-12.1(0.2)$ \\
\hline $2009 \mathrm{nr}^{\mathrm{e}}$ & $2010 \operatorname{Jan} 7.5$ & FAST & $55192.7(0.3)$ & $10.6(0.3)$ & $0.93(0.03)$ & $-9.3(0.1)$ & $-9.6(0.2)$ \\
\hline 2010A & 2010 Jan 16.1 & FAST & $55212.4(0.1)$ & $-0.3(0.1)$ & $0.88(0.02)$ & $-10.3(0.1)$ & $-10.3(0.2)$ \\
\hline
\end{tabular}

Notes. Uncertainties are listed in parentheses.

${ }^{\text {a }}$ Days since $B$ maximum.

b Velocity information originally presented by FSK11.

${ }^{c}$ Light-curve parameters were originally reported by Ganeshalingam et al. (2010).

d All measurements were originally reported by Foley et al. (2012).

e Light-curve parameters were originally reported by Khan et al. (2011).

increase. For the results presented here, we classify SN 1994D as Single; if SN 1994D is classified instead as Blueshifted, the significance of our results is reduced slightly, but not significantly.

\subsection{Phase}

The standard model to explain the variable $\mathrm{Na} \mathrm{D}$ seen in $\mathrm{SNe}$ Ia requires ionizing photons from the time of explosion (or immediately after) to ionize the $\mathrm{Na}$ atoms in the circumstellar environment (e.g., Patat et al. 2007b). After sufficient time, the $\mathrm{Na}$ atoms would recombine, resulting in increased $\mathrm{Na} \mathrm{D}$ absorption. If this model is correct, one might worry that systems with outflows will on average have no preferred line profile at early times (when the $\mathrm{Na}$ is ionized), but will on average have blueshifted profiles at later times.

To test this idea, we compared the different subsamples of the F12 sample. The F12 sample has a median phase of 1.0 day relative to $B$ maximum. The Blueshifted, Redshifted, Single/ Symmetric, and No Absorption subsamples have median phases of $-1.5,-2.3,15.5$, and 12.5 days, respectively. There is no indication that $\mathrm{SNe}$ in the Blueshifted subsample tend to have later phases.

There are three possible scenarios that allow for the Blueshifted subsample to predominantly come from progenitor systems with outflows and not have a phase bias. First, it is possible that the systems with variable $\mathrm{Na} \mathrm{D}$ are well tuned such that the ionizing flux and the distribution of the CSM are matched to show variability, while other systems with outflows simply do not ionize the CSM because of a lack of ionizing photons, the CSM being too far/dense, or both. Alternatively, it may be the case that the recombination timescale for most systems is shorter than the median time between explosion and the time of the spectrum, or about two weeks (although this is unlikely since variable $\mathrm{Na} \mathrm{D}$ is not seen until after maximum brightness even when there are earlier observations). Finally, progenitor systems may have $\mathrm{Na}$ at various radii from the $\mathrm{SN}$, where the closest $\mathrm{Na}$ is ionized, but the more distant Na produces blueshifted absorption. Indeed, the two SNe Ia with high-resolution spectroscopy and variable $\mathrm{Na} \mathrm{D}$ (SNe 2006X and 2007le) have blueshifted $\mathrm{Na} \mathrm{D}$ absorption at all spectroscopic epochs.
Regardless of why there is not an obvious phase bias in the F12 sample, it does not appear that the Blueshifted sample is missing a significant number of potential outflow systems specifically because the Na in the CSM was fully ionized at the time of spectroscopy.

\subsection{Light-curve Shape}

We would like to compare the decline rate distributions of the various samples. The full high-resolution sample, including all $\mathrm{SNe}$ Ia regardless of decline rate (i.e., the F12 sample combined with SNe Ia with high-resolution spectroscopy and $\Delta m_{15}(B)>1.5 \mathrm{mag}$ ), has a weighted mean $\Delta m_{15}(B)$ of $\left\langle\Delta m_{15}(B)\right\rangle=1.22 \pm 0.01 \mathrm{mag}$. The Blueshifted, Redshifted, Single/Symmetric, and No Absorption subsamples of the full high-resolution sample have weighted means of $\left\langle\Delta m_{15}(B)\right\rangle=$ $1.21 \pm 0.01,1.10 \pm 0.02,1.23 \pm 0.02$, and $1.45 \pm 0.03 \mathrm{mag}$, respectively. The subsample of SNe Ia with no $\mathrm{Na} D$ absorption has significantly faster light curves than the bulk of the sample. Six of $7 \mathrm{SNe}$ Ia in this subsample come from S0 or earlier host galaxies (Table 1), which is not surprising since these host galaxies are expected to have significantly less gas and dust, while no SN hosted in an elliptical galaxy is in any of the other subsamples (S11). SNe Ia in elliptical galaxies tend to have significantly faster light curves (e.g., Howell 2001; Hicken et al. 2009b).

The FSK11 method of determining $v_{\mathrm{Si} \text { II }}^{0}$ does not apply to $\mathrm{SNe}$ Ia with $\Delta m_{15}(B)>1.5 \mathrm{mag}$. As a result, $\mathrm{SNe}$ Ia with $\Delta m_{15}(B)>1.5$ mag cannot be included in the F12 sample and are therefore not included in any further analysis. Since many of the SNe Ia in the No Absorption subsample of the full highresolution sample have large decline rates, the F12 sample does not include many SNe Ia without Na D absorption. With this restriction on light-curve shape, the F12 sample has a weighted mean $\Delta m_{15}(B)$ of $\left\langle\Delta m_{15}(B)\right\rangle=1.14 \pm 0.01 \mathrm{mag}$, and the Blueshifted, Redshifted, Single/Symmetric, and No Absorption subsamples have weighted means of $\left\langle\Delta m_{15}(B)\right\rangle=1.12 \pm 0.01$, $1.10 \pm 0.02,1.23 \pm 0.02$, and $1.25 \pm 0.02 \mathrm{mag}$, respectively. The No Absorption subsample is still skewed to faster declining SNe relative to the entire F12 sample. 


\subsection{Maximum-light Color}

We now compare the $B_{\max }-V_{\max }$ pseudo-color (after correcting for Milky Way reddening) for the various samples. The FSK11 sample has a median pseudo-color of 0.01 mag. This is significantly bluer than the F12 sample, which has a median pseudo-color of $B_{\max }-V_{\max }=0.12 \mathrm{mag}$. A Kolmogorov-Smirnov (K-S) test results in a $p$-value of only 0.048 , indicating that the two samples may be drawn from different parent populations.

We interpret the color difference as a selection effect. Since the SN sample from which the FSK11 sample is derived is the sample of low- $z$ SNe discovered in SN surveys, there is some amount of Malmquist bias associated with both the discovery and follow-up of the sample. This bias will naturally remove extinguished SNe Ia near the limit of SN searches from the sample. However, for high-resolution spectroscopy, SNe typically need to be much brighter than the magnitude limit of SN searches; as a result, the Malmquist bias in the F12 sample should be significantly less than the FSK11 sample.

The median pseudo-colors for the Blueshifted, Redshifted, Single/Symmetric, and No Absorption subsamples are $B_{\max }-$ $V_{\max }=0.34,0.08,0.02$, and $0.00 \mathrm{mag}$, respectively. The values for the Blueshifted, Single/Symmetric, and No Absorption subsamples are fairly different from the median value for the full F12 sample. However, the Single/Symmetric and No Absorption subsamples have median pseudo-colors similar to that of the FSK11 sample, perhaps further indicating that $\mathrm{SNe}$ Ia in the Single/Symmetric and No Absorption subsamples have minimal dust extinction. Performing a K-S test between the Blueshifted subsample and the F12 sample excluding the Blueshifted subsample, we find a $p$-value of 0.021. A similar test for the Single/Symmetric and No Absorption subsamples results in $p$-values of 0.56 and 0.16 , respectively. Although the median values for all three subsamples suggest that there might be differences in the underlying populations of the subsamples, only the difference between the Blueshifted subsample and the remainder of the F12 sample is statistically significant.

The redder colors for the Blueshifted subsample are consistent with there being some SNe Ia in the sample with CSM resulting in additional reddening that is combined with the underlying host-galaxy reddening distribution.

\subsection{Ejecta Velocity}

With the knowledge that Blueshifted and No Absorption SNe Ia are redder and faster decliners than the rest of the F12 sample, respectively, while the other subsamples do not appear to be significantly different from each other, we now examine correlations between narrow $\mathrm{Na} \mathrm{D}$ profile properties and $v_{\mathrm{Si} \text { II }}^{0}$. Figure 2 displays the cumulative distribution function (CDF) of $v_{\mathrm{Si} \text { II }}^{0}$ for various samples. The gray and black lines represent the F12 and FSK11 samples, respectively. We also display the Blueshifted subsample of the F12 sample with a blue line.

The CDF of the F12 sample has a very similar shape to that of the FSK11 sample. The median $v_{\mathrm{Si} \text { II }}^{0}$ for both the F12 sample and the FSK11 samples is $-11,300$ and $-11,400 \mathrm{~km} \mathrm{~s}^{-1}$, respectively. Performing a K-S test (excluding any $\mathrm{SNe}$ in the F12 sample from the FSK11 sample), we find a K-S $p$-value of 0.87 . From this test, there is no indication that the F12 and FSK11 samples have different parent populations.

However, the Blueshifted subsample is qualitatively different from the FSK11 and F12 samples. Interestingly, the five SNe Ia with the highest $v_{\mathrm{Si} \text { II }}^{0}$ in the F12 sample are all in the Blueshifted

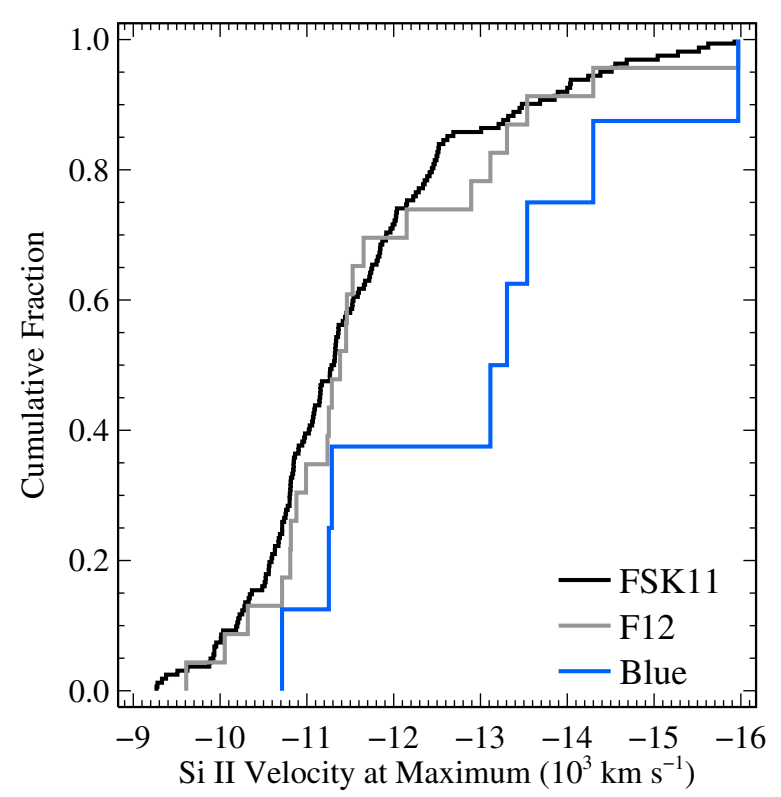

Figure 2. CDF of $v_{\mathrm{Si} \text { II }}^{0}$ for the FSK11 sample excluding $\mathrm{SNe}$ in the F12 sample (black line, labeled "FSK11"), the F12 sample (gray line, labeled "F12"), and the Blueshifted subsample (blue line, labeled "Blue").

(A color version of this figure is available in the online journal.)

subsample. Specifically, $63 \%$ of the Blueshifted subsample has a higher $v_{\mathrm{Si} \text { II }}^{0}$ than any SN Ia in any of the other subsamples. Not surprisingly, the CDF of the Blueshifted subsample appears to be significantly different from that of the FSK11 sample or the F12 sample, with the Blueshifted subsample tending to have higher $v_{\text {Si II }}^{0}$ than the typical SN Ia. The Blueshifted subsample has a median $v_{\mathrm{Si} \text { II }}^{0}$ of $-13,200 \mathrm{~km} \mathrm{~s}^{-1}$, while the Redshifted, Single/ Symmetric, and No Absorption subsamples have median $v_{\mathrm{Si} \text { II }}^{0}$ of $-11,300,-11,300$, and $-10,800 \mathrm{~km} \mathrm{~s}^{-1}$, respectively. A K-S test to compare the Blueshifted sample to the FSK11 sample (again, excluding any SNe Ia from the F12 sample) results in a $p$-value of 0.034 , indicating that the two samples likely have different parent distributions.

Although the F12 sample has a similar $v_{\mathrm{Si} \text { II }}^{0}$ distribution as the FSK11 sample, there may be other important ways in which the parent populations of the two samples are different. To mitigate any potential bias, we performed K-S tests between the Blueshifted subsample and the F12 sample (excluding the Blueshifted subsamples). This test resulted in a $p$-value of 0.018 . The Blueshifted subsample appears to be drawn from a different parent population than the complementary F12 sample.

We tested the robustness of this result using a Monte Carlo simulation. For each iteration, we selected a sample of $23 \mathrm{SNe}$ (to match the number of $\mathrm{SNe}$ Ia for which we have highresolution spectroscopy) from the full FSK11 sample of SNe Ia. From this sample, we then selected a subsample of "Blueshifted" $\mathrm{SNe}$ equal to the number of Blueshifted SNe Ia in the F12 sample. We performed a K-S test on these random samples similar to that described above. We also noted if the five highest or lowest velocity SNe were in the "Blueshifted" sample.

We found that $1.1 \%$ of all random samples had a $\mathrm{K}-\mathrm{S} p$-value as small or smaller than that of the Blueshifted sample. This value is similar to the $p$-value that we found above (0.018), and indicates that the $p$-value is a reasonable measurement of the probability of the Blueshifted and F12 samples being randomly drawn from the same parent distribution. We also found that $0.28 \%$ of the random samples had the five highest or lowest 


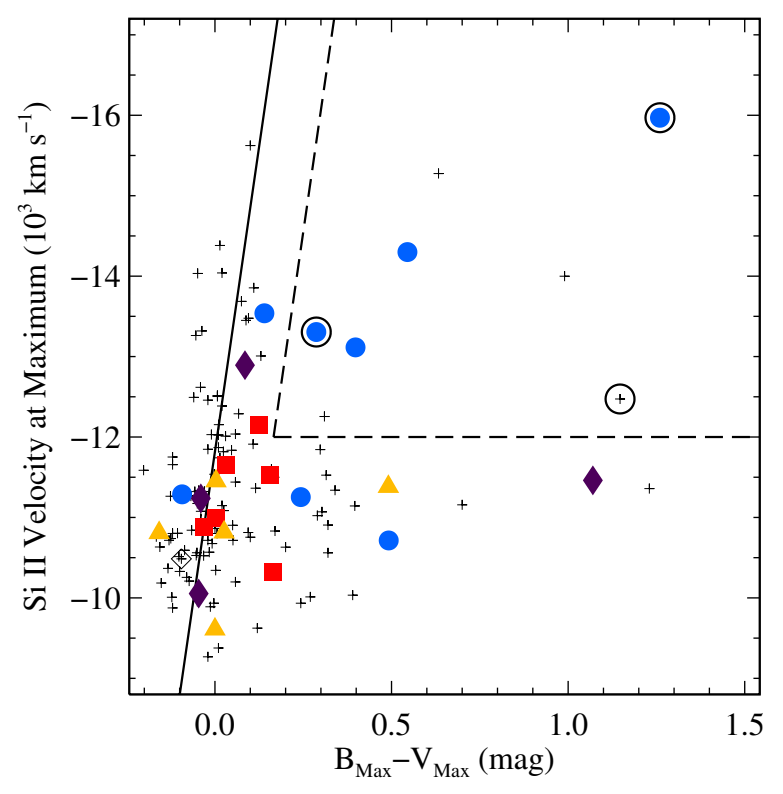

Figure 3. $v_{\mathrm{Si} \text { II }}^{0}$ as a function of $B_{\max }-V_{\max }$ pseudo-color for the FSK11 (black crosses), Blueshifted (blue circles), Redshifted (red squares), Single/Symmetric (purple diamonds), and No Absorption subsamples (gold triangles). The solid line is the velocity-color relation between $v_{S i_{I}}^{0}$ and intrinsic $B_{\max }-V_{\max }$ (FSK11). The empty black circles indicate SNe Ia with detected variable $\mathrm{Na}$ D. The region defined by the dashed lines (corresponding to a $2 \sigma$ deviation from the FSK11 relation between intrinsic $B_{\max }-V_{\max }$ and $v_{\mathrm{Si} \text { II }}^{0}$ and $v_{\mathrm{Si} \text { II }}^{0}=-12,000 \mathrm{~km} \mathrm{~s}^{-1}$ ) has a large fraction of SNe Ia with blueshifted and/or variable $\mathrm{Na} \mathrm{D}$ line profiles.

(A color version of this figure is available in the online journal.)

velocity SNe in the "Blueshifted" subsample. It is important to test both extremes since either case would have been notable to us. This test of the extreme $\mathrm{SNe}$ is perhaps a more interesting statistic since it is highly plausible that some of the Blueshifted systems come from the same parent population as the Redshifted systems, but are simply confused through random line-of-sight effects. Furthermore, only $0.21 \%$ of the random samples had both of the above characteristics. Although this last number is something of an a posteriori measurement, it does indicate that the number of extreme objects in the Blueshifted sample is providing additional information beyond the simple K-S statistic.

Compared to the F12 sample, the Blueshifted subsample appears to have a statistically significant difference in $v_{\mathrm{Si} \text { II }}^{0}$. As seen in Section 3.3, the SNe in the Blueshifted subsample also tend to have redder observed $B_{\max }-V_{\max }$ colors. Foley $\&$ Kasen (2011) and FSK11 showed that ejecta velocity and intrinsic color are highly correlated with $\mathrm{SNe}$ Ia with higher ejecta velocity tending to be intrinsically redder. In Figure 3, we show $v_{\mathrm{Si} \text { II }}^{0}$ as a function of observed $B_{\max }-V_{\max }$ for the FSK11 and F12 samples. We also display the FSK11 relation between intrinsic $B_{\max }-V_{\max }$ and $v_{\mathrm{Si} \text { II }}^{0}$. Several SNe Ia (including the majority of the Blueshifted subsample) fall far off to the red of the FSK11 relation, indicating that their very red colors are dominated by dust reddening rather than the trend with intrinsic color.

In the color-velocity parameter space, the Blueshifted subsample is even more distinct from the rest of the F12 sample. Performing a two-dimensional K-S test on the Blueshifted subsample, the resulting $p$-value of 0.023 is further indication of the Blueshifted subsample being a distinct subpopulation in this parameter space.
In Figure 3, we have also indicated the SNe Ia for which variable $\mathrm{Na} \mathrm{D}$ has been detected. All three have high $v_{\mathrm{Si} \text { II }}^{0}$ and $B_{\max }-V_{\max }$. Two of those three have a blueshifted $\mathrm{Na} \mathrm{D}$ profile, while the third does not have the high-resolution spectroscopy necessary to make that measurement. If one wants to choose $\mathrm{SNe}$ Ia which have a high probability of having variable $\mathrm{Na}$ $\mathrm{D}$, one might want to choose reddened $\mathrm{SNe}$ Ia with high ejecta velocities. We defined a region of this parameter space corresponding to high velocity $\left(v_{\mathrm{Si} \text { II }}^{0}<-12,000 \mathrm{~km} \mathrm{~s}^{-1}\right)$ and red (redder than the $2 \sigma$ deviation from the FSK11 relation between intrinsic $B_{\max }-V_{\max }$ and $v_{\mathrm{SiII}}^{0}$, which roughly corresponds to $B_{\max }-V_{\max }>0.2 \mathrm{mag}$ ). This region of parameter space contains half of the Blueshifted subsample and all SNe Ia with observed $\mathrm{Na} \mathrm{D}$ variability. We suggest that $\mathrm{SNe}$ Ia in this region are excellent candidates for having variable $\mathrm{Na} \mathrm{D}$. Additionally, special attention should be paid to $\mathrm{SNe}$ that have Blueshifted $\mathrm{Na} \mathrm{D}$ absorption in the first epoch.

\section{DISCUSSION AND CONCLUSIONS}

For the first time, we have shown a direct connection between the progenitor system of an SN Ia and its explosion properties. In contrast, previously, only indirect connections between progenitors and $\mathrm{SN}$ Ia properties have been made by examining host-galaxy properties (e.g., Hamuy et al. 1996; Howell 2001; Hicken et al. 2009b). Specifically, we find that $\mathrm{SNe}$ Ia with blueshifted narrow $\mathrm{Na} \mathrm{D}$ profiles tend to have higher velocity ejecta as probed by Si II $\lambda 6355$ at maximum brightness than those with no $\mathrm{Na} \mathrm{D}$ absorption or those with redshifted, single, or symmetric profiles. This was achieved by expanding the S11 sample of SNe Ia with additional SNe Ia with high-resolution spectroscopy and matching that expanded highresolution sample to the FSK11 sample of SNe Ia with measured $v_{\mathrm{Si} \text { II }}^{0}$, again expanding the FSK11 sample slightly with new data presented here. The resulting sample is designated the "F12" sample.

This result is further evidence that a significant fraction of SN Ia progenitor systems have outflows (S11), and that the large fraction of SNe Ia with blueshifted $\mathrm{Na} \mathrm{D}$ line profiles is not caused by some other effect. Similarly, this result supports the interpretation that the variable $\mathrm{Na} \mathrm{D}$ is the result of ionized CSM (Patat et al. 2007b; Simon et al. 2009) rather than a geometric effect (Chugai 2008; Patat et al. 2010).

For most subsamples and the larger SN Ia population, there is no indication of different light-curve shape distributions. However, the No Absorption subsample tends to have faster declining light curves (as expected given the host-galaxy distribution of the subsample). There is no phase bias for the Blueshifted subsample, indicating that few systems with outflows are artificially placed in other subsamples because the circumstellar $\mathrm{Na}$ is completely ionized at the time of spectroscopy.

The F12 sample has on average redder $B_{\max }-V_{\max }$ pseudocolors than the FSK11 sample. We suggest that this difference is the result of Malmquist bias reducing the number of reddened SNe in the FSK11 sample. Compared to the rest of the F12 sample, the Blueshifted subsample is slightly redder and the No Absorption subsample is slightly bluer. The bluer color for the No Absorption subsample is consistent with an SN population that has minimal dust reddening. Similarly, the redder color for the Blueshifted subsample is consistent with an SN population with additional dust in the circumstellar environment.

High-resolution spectra of $\mathrm{SNe}$ Ia in E/S0 galaxies have never shown $\mathrm{Na} \mathrm{D}$ absorption (with the exception of $\mathrm{SNe}$ Ia hosted in peculiar S0 galaxies, such SN 1986G, which was 
in Cen A). Most single-degenerate progenitor scenarios should exist in old stellar populations, so the different host properties for the different subsamples indicate that the population of SN progenitors with strong outflows may be extremely rare in old stellar populations. Alternatively, SNe Ia from early- and latetype galaxies may have, on average, very different progenitor systems. Future studies of SN hosts and locations may constrain the age of these progenitor systems, further constraining the exact progenitor systems.

There are clear implications for the connection between blueshifted $\mathrm{Na} \mathrm{D}$ absorption profiles and high ejecta velocities. Specifically, if all SNe Ia come from a single progenitor channel, then one requires an asymmetric progenitor system and explosion where the higher-velocity ejecta are aligned with the higher density regions of the CSM or explosions with higher kinetic energy per unit mass also have a denser and/or closer CSM. Alternatively, SNe Ia could come from a variety of progenitor channels where those that originate in progenitor systems with strong outflows tend to have more kinetic energy per unit mass than those with weak or no outflows.

From several different methods, specific progenitor systems which should have strong outflows have been ruled out for SN 2011fe (Li et al. 2011; Bloom et al. 2012), and there is strong evidence that its progenitor system had a clean environment (Chomiuk et al. 2012; Patat et al. 2011; Margutti et al. 2012). Since a significant fraction of SNe Ia do have strong progenitor outflows, it appears that there are at least two progenitor channels for SNe Ia.

R.J.F. is supported by a Clay Fellowship. A.G.Y is supported by grants from the ISF and Minerva foundations, an ARCHES award, and the Lord Sieff of Brimpton Fund. A.S. is supported by a Minerva Fellowship. M.H. acknowledges support by CONICYT through grants FONDECYT Regular 1060808, Centro de Astrofisica FONDAP 15010003, Centro BASAL CATA (PFB-06), and the Millenium Center for Supernova Science (P10-064-F). Supernova research at Harvard College Observatory is supported by NSF grant AST-0907903.

We are grateful to S. Blondin and M. Hicken, who reduced some of the data discussed in this work, and M. Shetrone, who assisted with the HET data reduction.. We thank P. Challis, A. Filippenko, and H. Marion for looking for old data. G. Folatelli helped organize the observing at Las Campanas and M. Stritzinger observed one low-resolution spectrum presented in this work. We appreciate comments from P. Nugent, F. Patat, L. Bildsten, A. Soderberg, and L. Chomiuk.

We are grateful to the staffs at the Las Campanas, McDonald, and Fred L. Whipple Observatories for their dedicated services. The HET is a joint project of the University of Texas at Austin, the Pennsylvania State University, Stanford University, Ludwig-Maximilians-Universität München, and Georg-AugustUniversität Göttingen. The HET is named in honor of its principal benefactors, William P. Hobby and Robert E. Eberly.

\section{REFERENCES}

Allington-Smith, J., Breare, M., Ellis, R., et al. 1994, PASP, 106, 983 Amanullah, R., Lidman, C., Rubin, D., et al. 2010, ApJ, 716, 712 Blondin, S., Dessart, L., Leibundgut, B., et al. 2006, AJ, 131, 1648 Blondin, S., Kasen, D., Röpke, F. K., Kirshner, R. P., \& Mandel, K. S. 2011, MNRAS, 417, 1280

Blondin, S., Matheson, T., Kirshner, R. P., et al. 2012, AJ, 143, 126

Blondin, S., Prieto, J. L., Patat, F., et al. 2009, ApJ, 693, 207
Bloom, J. S., Kasen, D., Shen, K. J., et al. 2012, ApJ, 744, L17

Brown, P. J., Dawson, K. S., de Pasquale, M., et al. 2011, arXiv:1110.2538

Burns, C. R., Stritzinger, M., Phillips, M. M., et al. 2011, AJ, 141, 19

Chomiuk, L., Soderberg, A. M., Moe, M., et al. 2012, ApJ, 750, 164

Chugai, N. N. 2008, Astron. Lett., 34, 389

Conley, A., Guy, J., Sullivan, M., et al. 2011, ApJS, 192, 1

D’Odorico, S., di Serego Alighieri, S., Pettini, M., et al. 1989, A\&A, 215, 21

Dressler, A., Hare, T., Bigelow, B. C., \& Osip, D. J. 2006, Proc. SPIE, 6269, $62690 \mathrm{~F}$

Edwards, Z. I., Pagnotta, A., \& Schaefer, B. E. 2012, ApJ, 747, L19

Fabricant, D., Cheimets, P., Caldwell, N., \& Geary, J. 1998, PASP, 110, 79

Foley, R. J., Challis, P. J., Filippenko, A. V., et al. 2012, ApJ, 744, 38

Foley, R. J., \& Kasen, D. 2011, ApJ, 729, 55

Foley, R. J., Papenkova, M. S., Swift, B. J., et al. 2003, PASP, 115, 1220

Foley, R. J., Rest, A., Stritzinger, M., et al. 2010, AJ, 140, 1321

Foley, R. J., Sanders, N. E., \& Kirshner, R. P. 2011, ApJ, 742, 89

Freedman, W. L., Burns, C. R., Phillips, M. M., et al. 2009, ApJ, 704, 1036

Gamezo, V. N., Khokhlov, A. M., \& Oran, E. S. 2005, ApJ, 623, 337

Ganeshalingam, M., Li, W., Filippenko, A. V., et al. 2010, ApJS, 190, 418

Hamuy, M., Folatelli, G., Morrell, N. I., et al. 2006, PASP, 118, 2

Hamuy, M., Phillips, M. M., Suntzeff, N. B., et al. 1996, AJ, 112, 2391

Hicken, M., Challis, P., Jha, S., et al. 2009a, ApJ, 700, 331

Hicken, M., Wood-Vasey, W. M., Blondin, S., et al. 2009b, ApJ, 700, 1097

Hillebrandt, W., \& Niemeyer, J. C. 2000, ARA\&A, 38, 191

Ho, L. C., \& Filippenko, A. V. 1995, ApJ, 444, 165

Horne, K. 1986, PASP, 98, 609

Howell, D. A. 2001, ApJ, 554, L193

Howell, D. A. 2011, Nat. Commun., 2, 350

Hoyle, F., \& Fowler, W. A. 1960, ApJ, 132, 565

Iben, I., Jr., \& Tutukov, A. V. 1984, ApJS, 54, 335

Iben, I., Jr., \& Tutukov, A. V. 1996, ApJS, 105, 145

Kessler, R., Becker, A. C., Cinabro, D., et al. 2009, ApJS, 185, 32

Khan, R., Prieto, J. L., Pojmański, G., et al. 2011, ApJ, 726, 106

Khokhlov, A. M. 1991, A\&A, 245, 114

King, D. L., Vladilo, G., Lipman, K., et al. 1995, A\&A, 300, 881

Leonard, D. C., Li, W., Filippenko, A. V., Foley, R. J., \& Chornock, R. 2005, ApJ, 632,450

Li, W., Bloom, J. S., Podsiadlowski, P., et al. 2011, Nature, 480, 348

Li, W., Filippenko, A. V., Gates, E., et al. 2001, PASP, 113, 1178

Maeda, K., Benetti, S., Stritzinger, M., et al. 2010, Nature, 466, 82

Maeda, K., Leloudas, G., Taubenberger, S., et al. 2011, MNRAS, 413, 3075

Maoz, D., \& Mannucci, F. 2008, MNRAS, 388, 421

Margutti, R., Soderberg, A. M., Chomiuk, L., et al. 2012, arXiv:1202.0741

Mazzali, P. A., Röpke, F. K., Benetti, S., \& Hillebrandt, W. 2007, Science, 315, 825

Nomoto, K., Thielemann, F.-K., \& Yokoi, K. 1984, ApJ, 286, 644

Nugent, P. E., Sullivan, M., Cenko, S. B., et al. 2011, Nature, 480, 344

Pakmor, R., Kromer, M., Röpke, F. K., et al. 2010, Nature, 463, 61

Patat, F., Benetti, S., Cappellaro, E., et al. 1996, MNRAS, 278, 111

Patat, F., Benetti, S., Justham, S., et al. 2007a, A\&A, 474, 931

Patat, F., Chandra, P., Chevalier, R., et al. 2007b, Science, 317, 924

Patat, F., Chandra, P., Chevalier, R., et al. 2011, arXiv:1112.0247

Patat, F., Cox, N. L. J., Parrent, J., \& Branch, D. 2010, A\&A, 514, A78

Perlmutter, S., Aldering, G., Goldhaber, G., et al. 1999, ApJ, 517, 565

Rich, R. M. 1987, AJ, 94, 651

Riess, A. G., Filippenko, A. V., Challis, P., et al. 1998, AJ, 116, 1009

Riess, A. G., Strolger, L.-G., Casertano, S., et al. 2007, ApJ, 659, 98

Schaefer, B. E., \& Pagnotta, A. 2012, Nature, 481, 164

Silverman, J. M., Foley, R. J., Filippenko, A. V., et al. 2012, arXiv:1202.2128

Sim, S. A., Röpke, F. K., Hillebrandt, W., et al. 2010, ApJ, 714, L52

Simon, J. D., Gal-Yam, A., Gnat, O., et al. 2009, ApJ, 702, 1157

Simon, J. D., Gal-Yam, A., Penprase, B. E., et al. 2007, ApJ, 671, L25

Sollerman, J., Cox, N., Mattila, S., et al. 2005, A\&A, 429, 559

Sternberg, A., Gal-Yam, A., Simon, J. D., et al. 2011, Science, 333, 856

Stritzinger, M. D., Phillips, M. M., Boldt, L. N., et al. 2011, AJ, 142, 156

Suzuki, N., Rubin, D., Lidman, C., et al. 2012, ApJ, 746, 85

Tull, R. G. 1998, Proc. SPIE, 3355, 387

van Kerkwijk, M. H., Chang, P., \& Justham, S. 2010, ApJ, 722, L157

Wade, R. A., \& Horne, K. 1988, ApJ, 324, 411

Wang, B., \& Han, Z. 2012, arXiv: 1204.1155

Wang, X., Li, W., Filippenko, A. V., et al. 2008, ApJ, 675, 626

Webbink, R. F. 1984, ApJ, 277, 355

Whelan, J., \& Iben, I. J. 1973, ApJ, 186, 1007

Wood-Vasey, W. M., Miknaitis, G., Stubbs, C. W., et al. 2007, ApJ, 666, 694

Woosley, S. E., Taam, R. E., \& Weaver, T. A. 1986, ApJ, 301, 601 